\title{
Solodyn and Updates on Topical and Oral Therapies for Acne
}

\author{
Whitney P. Bowe • Jaimie B. Glick • Alan R. Shalita
}

Published online: 17 June 2012

(C) Springer Science+Business Media, LLC 2012

\begin{abstract}
Acne vulgaris is a chronic and ubiquitous skin condition that can result in both physical and psychological scarring. The need for early and aggressive treatment of acne is essential to prevent these deleterious effects. Understanding of acne pathophysiology continues to evolve, leading to new therapeutic targets and the development of advanced treatment regimens. Among the most recent advances in acne therapy are new fixed-drug combination therapies and novel vehicle technologies. Additionally, as the concern for antibiotic resistance grows, treatments involving nonantibiotic therapies are growing in popularity. Dermatologists have also begun to recognize the importance of diet effects on acne progression, as well as the therapeutic role of procedural acne treatments. This review summarizes the most recent developments in acne pathophysiology and discusses new trends in therapy.
\end{abstract}

Keywords Dermatology · Dermapharmacology · Therapeutic development · Acne · Pathophysiology ·

Treatment guidelines · Topical acne treatment . Combination topical therapy · Innovative drug delivery systems . Formulation - Antibiotics - Antibiotic resistance - Diet . Light $\cdot$ Laser $\cdot$ Photopneumatic therapy $\cdot$ Retinoid . Nanoparticles $\cdot$ Microspheres

W. P. Bowe $(\triangle) \cdot$ J. B. Glick $\cdot$ A. R. Shalita Department of Dermatology, SUNY Downstate Medical Center, 450 Clarkson Ave, Brooklyn, NY, USA

e-mail: wpbowe@gmail.com

\section{Introduction}

Acne vulgaris is one of the most common cutaneous disorders, affecting more than $80 \%$ of adolescents and frequently persisting into adulthood [1]. Acne is a chronic condition of the pilosebaceous unit (PSU), which is characterized by inflammatory lesions (papules, pustules, and nodules) and noninflammatory lesions (open and closed comedones). In 2009, the Global Alliance to Improve Outcomes in Acne published an update on the management of acne vulgaris, emphasizing the importance of viewing acne as a chronic disease $[2 \bullet \bullet$. Because of its chronicity, acne is treated over prolonged periods of time, and patients often suffer from long-term physical and psychological effects. Thus, the expert panel comprising the Alliance encouraged early and aggressive acne treatment.

This review begins with a discussion of the most recent updates in acne pathophysiology. The latest advances in acne therapy-including new fixed-drug combinations, advanced treatment formulations, and novel medications - are subsequently addressed. Finally, insights into the influence of diet on acne and the efficacy of procedural treatments for acne are offered.

\section{Pathophysiology of Acne: The Old and The New}

The pathogenesis of acne is multifactorial and has been attributed to four primary pathogenic factors: excess sebum production, follicular colonization by Propionibacterium acnes (P. acnes), alteration in keratinization, and inflammation [3]. Traditionally, the microcomedo was believed to be the primary lesion in acne, which later developed into an inflammatory or noninflammatory lesion. The formation of comedones was believed to result from failure of 


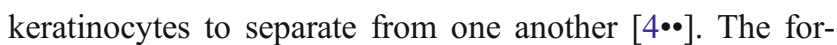
mation of the keratin plug and increased sebum production was then thought to be followed by colonization with $P$. acnes, and ultimately induction of the innate immune response leading to inflammation. P. acnes appears to activate the inflammatory cascade through Toll-like receptors (TLRs), specifically TLR-2 [5]. However, recent evidence has called this traditionally accepted sequence of events into question. This evidence suggests that inflammation may precede these other events. Jeremy et al. [6] suggest inflammation may precede keratinization and noted the presence of elevated levels of proinflammatory cytokine interleukin (IL)-1, even prior to follicular plugging. Additionally, new data suggests that the secretion of a biofilm by $P$. acnes may act as an "adhesive glue," facilitating comedogenesis [7]. Treatment targeting the $P$. acnes biofilm is likely the target of photopneumatic therapy, a procedural technique used in acne treatment (see "Photopneumatic Therapy").

New insights into the mechanism of activation of the androgen receptor and subsequent sebum production have also been proposed. It has long been known that androgenic hormones stimulate androgen receptors, ultimately leading to increased sebum production. Melnik $[8,9 \cdot \bullet, 10,11]$ postulates that the removal of the polypeptide nuclear transcription factor Fox01 "opens" the androgen receptor so that it can be accessed by androgenic agonists. It is the phosphorylation of Fox01 that facilitates its removal from the androgen receptor. It is thought that increased levels of IGF1 and insulin mediate this phosphorylation $[8,9 \cdot \bullet, 10,11]$. Certain dairy products and high-glycemic load diets are thought to play a role in enhancing this mechanism.

New data suggests that the sebaceous gland is regulated by neuropeptides and can serve as an independent neuroendocrine organ $[12 \bullet \cdot]$. The corticotrophin-releasing hormone $(\mathrm{CRH})$ is activated by proinflammatory cytokines in response to stress [13]. CRH and CRH receptor (CRH-R) were found in SZ95 sebocytes in vitro and human sebaceous glands in situ $[14,15]$, suggesting a link between CRH, stress, and acne. A CRH-R antagonist known as alpha-helical CRF was found to block the effects of CRH on the SZ95 sebocyte, thereby elucidating a role for the development of $\mathrm{CRH}$ antagonists in the treatment of acne [16]. Moreover, melanocortins and their receptors have been identified in human skin and may prove to be clinically useful for the treatment of excessive sebum production in acne [12••, 17-19]. The response of skin to stress and its relationship to neuroendocrine function may soon present new targets for therapeutic interventions.

\section{Current Treatment Guidelines}

The Global Alliance to Improve Outcomes in Acne created recommendations for acne treatment in 2003 [20] and published an update in 2009 [2••]. Acne treatment is based on the graded severity of acne lesions from mild to moderate to severe acne. The Alliance supports the use of fixed-dose topical combination products, as they increase efficacy by targeting multiple pathogenic factors and promote increased patient adherence. The report suggests using topical retinoids in all patients with acne except those with the most severe cases. When inflammatory lesions are present, a topical retinoid should be used in combination with an antimicrobial agent such as benzoyl peroxide (BPO) or an antibiotic. Based on published data, the Alliance suggests the limitation of oral antibiotic therapy to a period of less than 3-4 months and maintenance therapy using a topical retinoid or a topical retinoid-antibacterial combination.

The Alliance also recognizes the role of antibiotic resistance in acne management. It acknowledges the need to limit both the frequency and duration of antibiotic use. When long-term antibiotic use is necessary, the Alliance recommends adding a nonantibiotic antimicrobial agent like BPO. Unlike antibiotics, BPO has not been associated with bacterial resistance.

The 2009 update presents an in-depth discussion of the role of procedural treatments in acne $[2 \bullet \bullet$. The report addresses the potential benefit of these techniques but emphasizes the need for randomized controlled trials (RCTs) to further investigate the efficacy and short- and long-term effects of treatment.

\section{Advances in Topical Acne Treatment}

Topical therapies are the mainstay of treatment for mild to moderate acne and are used as maintenance therapy for all levels of acne. Retinoids (adapalene, tazarotene, tretinoin) and retinoid-like medications (salicylic acid) have comedolytic effects, and act to reverse abnormal desquamation [21]. Retinoids have also been shown to modulate the inflammatory response associated with acne [22]. Topical antibiotics and $\mathrm{BPO}$ are antibacterial agents that inhibit the proliferation of $P$. acnes [23-25]. Acne treatment often requires the use of several of these medications to address the multifactorial nature of the disorder $[2 \bullet \bullet, 20]$. Although effective, topical medications are associated with several side effects - including dryness, irritation, and scaling-that compromise patient compliance and ultimately decrease therapeutic efficacy. New combinations and formulations of topical treatments have been developed to contend with these concerns.

\section{Combination Topical Therapy}

Dermatologists and researchers are continuously investigating optimal drug combinations in the treatment of acne vulgaris. A PubMed search for RCTs of topical combination 
acne medications using the keywords topical acne treatment, acne therapy, combination acne treatment, and topical combination acne was performed for the period of December 2010 to December 2011 (Table 1).

Fixed-dose combinations of acne treatment represent the new direction for acne therapy, as they promote patient adherence and subsequently improve efficacy. The most recent RCTs involving fixed-dose combination therapy are marked by an “*” in Table 1. Studies have demonstrated that simplifying treatment regimens could lead to improved adherence and outcomes with topical acne medication [26•]. Twenty-six patients with mild to moderate acne were randomized to receive a fixeddose combination clindamycin phosphate $1.2 \%$-tretinoin $0.025 \%$ gel (CTG) known as Ziana ${ }^{\circledR}$ (Medicis, Scottsdale, Arizona), or a generic clindamycin phosphate gel $1 \%(\mathrm{C}$ gel) and a separate tretinoin cream $0.025 \%$ (T cream) to apply to the entire face. Adherence to treatment was monitored with the Medication Event Monitoring System ${ }^{\circledR}$ (AARDEX Group, Sion, Switzerland), which monitors when the medication was opened. A statistically significant difference in adherence between groups was noticed at 12 weeks $(P<0.02)$ with the CTG group demonstrating greater adherence. Both groups had significant improvement in inflammatory and total lesion counts $(P<0.05)$ by 12 weeks. However, the CTG group showed a trend toward greater improvement from baseline than the $\mathrm{C}$ gel $+\mathrm{T}$ cream group at each time point in the study, even though statistical significance was not reached. The authors suggest the lack of statistical significance may be a result of the small sample size and the likelihood that patients will reduce the use of treatments as their condition improves [26•]. Another combination therapy that has improved medication compliance in the treatment of acne is adapalene $0.1 \%$ and BPO $2.5 \%$ (Epiduo $^{\circledR}$, Galderma, Fort Worth, Texas), which is further discussed in the section "Microspheres."

\section{Innovative Drug Delivery Systems}

New drug delivery systems for topical medications have moved to the forefront of acne research. Drug delivery systems such as liposomes, microspheres, and solid lipid nanoparticles (SLNs) enhance follicular targeting to allow high concentrations of the active drug to penetrate the PSU [27-29]. Additionally, these systems allow for controlled release and gradual distribution of therapy, leading to reduced irritancy and side effects.

\section{Liposomes}

Liposomes are among the first generation of the novel drug delivery systems. They are spherical vesicles formed of one or more concentric phospholipid bilayers containing a central aqueous cavity [30•]. The biphasic nature of liposomes allows for carriage of hydrophobic and lipophilic medications. The use of drug-loaded liposomes for the treatment of acne has been widely reported [30॰]. A recent double-blind clinical trial compared the safety and efficacy of liposome-encapsulated $1 \%$ clindamycin lotion to $1 \%$ clindamycin solution [31]. At the end of 6 weeks, patients treated with the liposomeencapsulated $1 \%$ clindamycin lotion showed a $33.3 \%$ decrease in open comedones versus only a $8.3 \%$ decrease in the commercial clindamycin lotion. Reports have also indicated the effectiveness of liposomal derivatives of tea tree oil, salicylic acid, retinoids, and BPO in acne treatment [30•].

\section{Microspheres}

Microsphere is a vehicle technology comprised of inert particles made of synthetic polymers designed to entrap an active ingredient [32]. The particles protect the entrapped compound from degradation and are generally more stable than liposomes. The technology allows for prolonged drug delivery that leads to increased transdermal penetration without overloading of the epidermis [32]. Consequently, less product is left on the skin surface, leading to reduced irritancy and side effects. In addition, microsphere formulations have enhanced the stability of tretinoin, a particularly unstable molecule that can be degraded when used in combination with BPO or when exposed to UV radiation [33, 34]. Although microsphere technology has been available in acne treatment for over a decade, research continues to improve on this technology.

In a recent study $0.04 \%$ tretinoin microsphere gel was associated with fewer episodes of dryness and erythema than $0.1 \%$ tretinoin microsphere gel [35]. Both gels represent different concentrations of the product Retin-A Micro ${ }^{\circledR}$ microsphere (Johnson \& Johnson, New Brunswick, New Jersey). The $0.04 \%$ tretinoin gel was also formulated into a pump and evaluated in a blinded, randomized split-face study of 170 acne patients. Results showed a decrease in the irritation potential of $0.04 \%$ tretinoin microsphere gel in a pump form when compared to adapalene $0.1 \%+$ BPO $2.5 \%$ [36]. The pump microsphere formulation was associated with significantly less erythema, burning, and itching than the adapalene + BPO combination.

Microsphere technology has also proved beneficial in the design of BPO creams and washes. In an investigatorblinded, randomized, multicenter study comparing BPO $5.5 \%$ microsphere cream $\left(\mathrm{NeoBenz}{ }^{\circledR}\right.$ Micro, SkinMedica, Carlsbad, California) to BPO $6 \%$ gel, the microsphere cream demonstrated a greater reduction in noninflammatory and total lesion counts, although the results were not statistically significant [37]. Tolerability scores were significantly better for the BPO microsphere cream than the standard gel. Microspheres have also been effective in treating "facial shine," an oily appearance to the skin, which is a common 


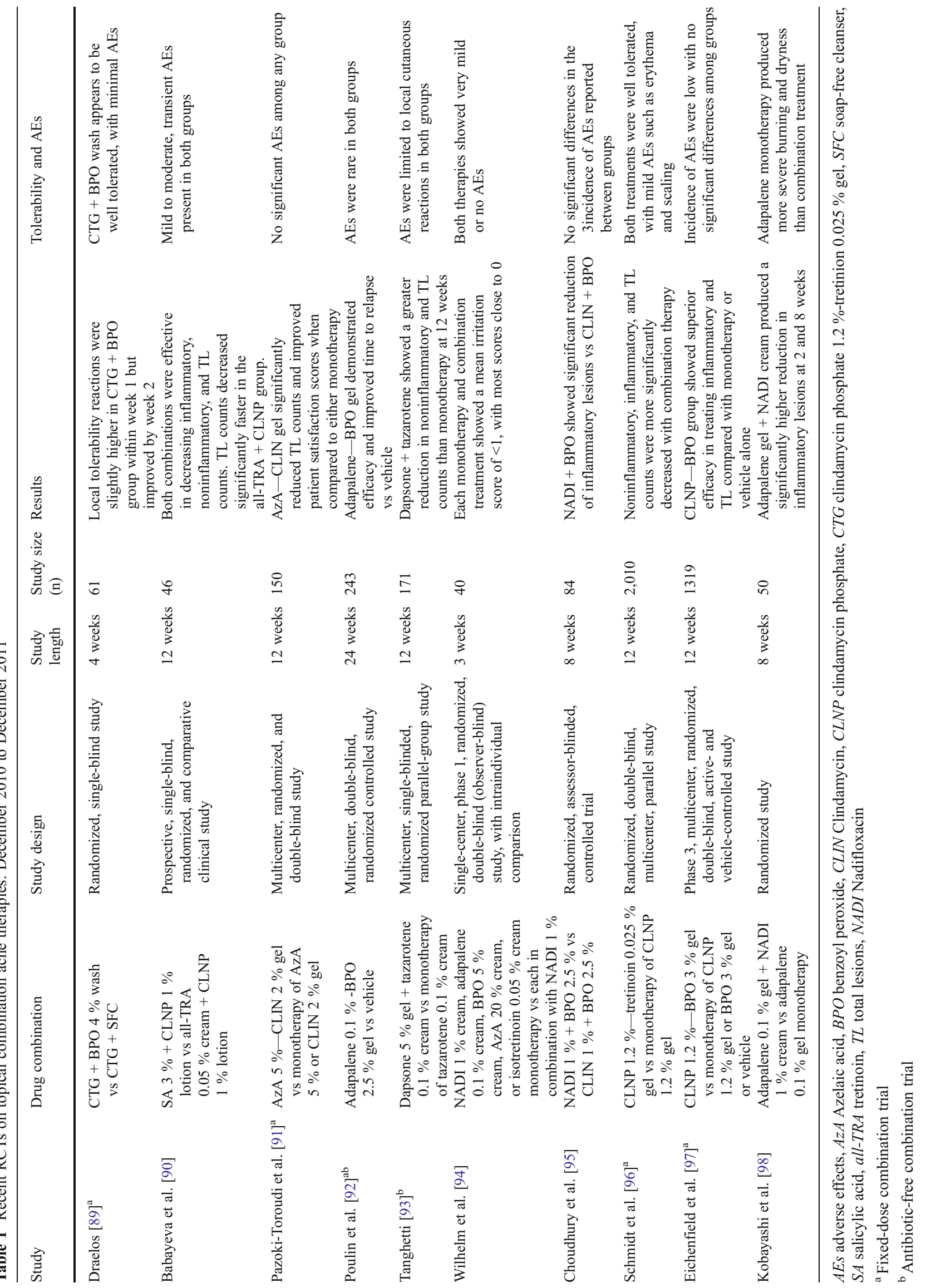


concern for many acne patients. Microsphere formulations of BPO and tretinoin were shown in separate split-face studies to reduce facial shine and associated sebum accumulation in the skin of acne patients [38•, 39].

\section{Solid Lipid Nanoparticles}

SLNs are solid, submicronic particles capable of carrying lipophilic and hydrophilic drugs [40]. Initially, the encapsulation of retinoids into SLN was a challenge due to retinoid instability. However, several recent advances have improved on this technology. A study published in 2011 used ion pairing between all-trans retinoic acid (atRA) and a lipophilic amine to improve the encapsulation process. In a rhino mouse model, the ion paired atRA-loaded SLNs demonstrated similar comedolytic effects and epidermal thickening to both $0.01 \%$ atRA gel and $0.05 \%$ atRA cream [41•]. Furthermore, the RA-loaded SLN more significantly reduced RA-induced erythema and scaling when compared to the conventional formulations.

Another encapsulation process using chitosan is being investigated. Chitosan is a biopolymer with bioadhesive and antibacterial activity [42]. A recent in vitro study of RA-chitosan loaded SLN demonstrated high physical stability over a 1-year period, high encapsulation efficiency, and high antibacterial activity against $P$. acnes [42]. Research into the use of SLNs is ongoing, and dermatologists are likely to see the development of even more cutting-edge technologies in the years to come.

\section{Unique Formulations}

Particle size plays a key role in effective acne treatment as smaller particles can better penetrate the PSU. Recently, a micronized formulation of $0.05 \%$ tretinoin gel, known as Atralin ${ }^{\circledR}$ (Coria Laboratories, Fort Worth, Texas), has become available [43]. This low-concentration medication is comprised of micronized particles, with most of the particles less than $10 \mu \mathrm{m}$. The particles are also suspended in a hydrogel, allowing for regulated drug release and enhanced moisturizing capacity. [43] A post hoc analysis of 299 adolescents with mild to moderate acne compared the efficacy and tolerability of $0.05 \%$ micronized tretinoin gel to $0.1 \%$ tretinoin gel microsphere [43]. The micronized tretinoin gel and tretinoin gel microsphere demonstrated comparable lesion reduction, but micronized tretinoin gel displayed enhanced tolerability.

A nonprescription product combining a micronized 5.5\% BPO and $0.4 \%$ micro-exfoliating lipophilic derivative of salicylic acid (LHA), known as Effaclar Duo ${ }^{\circledR}$ (La RochePosay, France), has recently come to the market. The micronized BPO particles are designed to penetrate deep into the PSU with limited exposure to the skin surface, thereby theoretically decreasing irritation. Salicylic acid is an effective comedolytic agent, and in vitro studies show this new LHA derivative has six times the keratolytic action of salicylic acid.

Azelaic acid (AzA) is a naturally occurring dicarboxylic acid with anti-inflammatory and antimicrobial effects that has been used for several decades in the treatment of acne. Recently, a new formulation of AzA complexed with niacinamide and glycerin, known as AzaClear ${ }^{\circledR}$ (Epikinetics Pharma, Los Angeles, California), has been developed for the treatment of acne vulgaris. Niacinamide appears to enhance treatment effects, whereas glycerin acts as an emollient.

A novel drug formulation, APDDR-0901, was recently developed in Korea to improve the side effects and efficacy of retinol. A $0.03 \%$ retinol was complexed with a $0.7 \%$ rose extract and a $0.05 \%$ hexamidine diisethionate cream. Hexamidine diisethionate has been shown to be active against $P$. acnes [44], whereas rose extract is thought to have anti-inflammatory effects. A double-blind RCT compared the APDDR-0901 formulation to $0.1 \%$ adapalene gel [45]. Results showed similar efficacy between medications. However, the APDDR-0901 demonstrated significantly fewer cases of erythema, scaling, and burning than the adapalene gel.

Acne located on the back or chest is referred to as truncal acne and is a common presentation of acne vulgaris [46]. Truncal acne can be challenging to treat, as the large surface area makes topical medications more difficult to apply [46]. In a recent study, a BPO $5.3 \%$ emollient foam (BenzEFoam ${ }^{\circledR}$, Onset Therapeutics, Cumberland, Rhode Island) was evaluated in patients with $P$. acnes on their backs [46]. Twenty subjects were treated once daily with BPO $5.3 \%$ emollient foam for 2 weeks and received no treatment in week 3 . In the last 2 weeks of the study, patients used a BPO $8 \%$ wash on their backs. P. acnes cultures were taken after each week, with results showing a significant reduction in $P$. acnes counts after the first 2 weeks of the BPO $5.3 \%$ emollient foam. There was no change in $P$. acnes counts in the last 2 weeks when the BPO wash was used. The authors suggest the emollient foam acts as a "leave-on" formula that permits longer contact between the skin and BPO with increased penetration of the PSU [46]. Of note, however, there are a number of cleansers that demonstrate the property of "substanstivity," meaning that BPO can persist on the skin long after the formula has been rinsed off.

Combination drug treatments, novel drug delivery systems, and unique formulations provide new and exciting alternatives to topical acne treatment. Many of these recent additions to dermatologists' acne armamentarium target multiple pathophysiologic processes in acne, reduce the complexity of treatment regimens, and improve tolerability. Novel drug combinations and 
formulations will continue to develop as new advances in vehicle technology are identified.

\section{Systemic Antibiotics}

Systemic antibacterials are indicated for the treatment of moderate to severe inflammatory acne and for patients who do not respond to topical acne treatments[2••, 47, 48]. Oral antibiotics are commonly administered in combination with topical medications like a retinoid and/or BPO to enhance therapeutic efficacy and decrease emergence of $P$. acnes resistance. Tetracyclines are the antibiotic class prescribed most frequently for the treatment of acne. Tetracyclines are bacteriostatic, and their mechanism of action involves the inhibition of $P$. acnes proliferation within the sebaceous follicles, as well as an independent antiinflammatory effect $[6,49-51]$. Of the tetracylines, doxcycline and minocycline are used most often because of their increased efficacy and decreased association with $P$. acnes resistance [52].

Minocycline is the most lipophilic of all the tetracyclines, thus increasing its ability to penetrate the lipid-rich sebaceous follicles [53]. The lipophilic nature of minocycline also enhances its absorption, making its ingestion less cumbersome than other tetrayclines, as it is less affected by food or dairy intake [54•]. Minocycline has also been associated with decreased bacterial resistance as compared to the other tetracyclines [55, 56]. Minocycline's enhanced lipophilicity not only improves its efficacy in acne treatment but also increases its ability to cross the blood-brain barrier leading to acute vestibular adverse events [57]. Solodyn ${ }^{\circledR}$ (Medicis, Scottsdale, Arizona) is an extended-release formulation of minocycline that has been able to decrease the existence of these adverse effects while maintaining the efficacy of minocycline immediate release. Solodyn's reduction in adverse drug reactions is related to its delayed time to peak plasma concentration (Tmax) and reduced peak plasma concentration (Cmax) [53]. Rapid absorption and/or high-peak concentrations of a medication may induce or exacerbate adverse drugs reactions [58]. Studies assessing the weight-based dosing of Solodyn demonstrate that a dose of $1 \mathrm{mg} / \mathrm{kg} / \mathrm{day}$ was as effective as $2 \mathrm{mg} / \mathrm{kg}$ per day and $3 \mathrm{mg} / \mathrm{kg}$ per day with a significant decrease in adverse effects, especially acute vestibular effects. [57, 59]. In 2006, the Food and Drug Administration (FDA) approved Solodyn for the treatment of nonnodular moderate to severe acne.

Some tetracylines are associated with other adverse reactions, including gastrointestinal distress and vaginal candidiasis [60•]. To prevent vaginal yeast infections, the authors suggest eating yogurt with live cultures or taking probiotics throughout the course of antibiotic treatment. Probiotics not only ameliorate the side effects of antibiotics but may decrease systemic levels of inflammation and regulate inflammatory cytokines in the skin [61]. Minocycline has also been associated with an increased risk of hyperpigmentation and systemic immunologic reactions including hypersensitivity and lupus-like syndromes with several reports present in the literature [54•]. It is unclear as of yet what Solodyn's role may be in exacerbating or reducing these specific adverse drug reactions [54•].

\section{Antibiotic Resistance: A Growing Concern}

Antibiotics used to be at the forefront of acne treatment and frequently used as monotherapy. However, antibiotic resistance has resulted in a decline in their therapeutic efficacy. Long-term use of antibiotics (both systemic and topical) produces selection pressure, which leads to the proliferation of antibiotic-resistant bacteria [62・•]. Long-term antibiotic use not only translates into decreased efficacy but also farreaching deleterious effects such as an increased risk of developing infections [62••]. Margolis et al. [63] showed that the odds of an upper respiratory tract infection in a patient receiving long-term antibiotics for acne was 2.15 times greater than those acne patients not receiving antibiotics. Furthermore, in a recent prospective study, patients receiving oral antibiotics for acne had three times the odds of developing pharyngitis than patients who were not taking antibiotics [64].

Long-term antibiotic use in acne has also been associated with the development of resistance in bacteria other than $P$. acnes, including coagulase-negative staphylococci (CNS) $[65,66]$. This is particularly concerning as antibioticresistant CNS can serve as a reservoir of antibiotic resistance genes and spread to other bacteria and hosts $[62 \bullet \bullet, 67]$. It is possible for these antibiotic-resistant genes to be transferred to more severe pathogenic organisms such as Staphylococci aureus (S. aureus) [62・•, 68]. These findings suggest the importance of limiting the use of antibiotics to the shortest duration and dosage necessary, and using combination therapy with BPO to slow the development of resistance.

Because of the growing concern of antibiotic resistance, antibiotic-free therapeutic options for the treatment of acne are increasing in popularity. Among the trials listed in Table 1, two demonstrate the effectiveness of antibiotic-free topical combination treatments. One of these studies includes a fixed dose combination of the adapalene $0.1 \%$ and BPO $2.5 \%$ (Epiduo ${ }^{\circledR}$, Galderma, Fort Worth, Texas). Although, BPO is effective against proliferation of $P$. acnes, there has been no evidence to date of $P$. acnes resistance to BPO. A 2011 openlabel, single-center study by Leyden et al. [69•] demonstrated the effectiveness of adapalene $0.1 \%$ and BPO $2.5 \%$ gel on antibiotic-sensitive and resistant $P$. acnes. In fact, the combination gel reduced strains of antibiotic-resistant and antibioticsensitive bacteria by $97 \%$. The decreased therapeutic efficacy 
of antibiotics against resistant $P$. acnes, as well as the global health impact of antibiotic resistance, makes nonantibiotic medications enticing options for dermatologists.

\section{Novel Topical and Oral Acne Medications}

\section{Topical Dapsone}

Among the most novel drugs available for acne treatment is dapsone $5 \%$ gel. Oral dapsone is an anti-inflammatory and antimicrobial medication used for the treatment of a number of skin disorders, but its increased side effect profile prevented its use in acne treatment [70•]. A topical form of dapsone has recently been approved for the treatment of acne vulgaris, based on two randomized, vehiclecontrolled studies [71]. Topical dapsone was also found to have a desirable side effect profile over the long-term in a 12-month safety and efficacy trial [72]. Moreover, the pharmacokinetics of the gel were examined in two separate studies [73]. Topical dapsone has been extensively studied and its safety confirmed; however, its efficacy appears to be modest in recent clinical trials. In practical application, dapsone appears to reduce the irritation of topical retinoids and contributes significantly to their efficacy.

\section{Oral Antifungals: A New Direction for Acne Treatment?}

P. acnes is not the only organism present in acne. In an article published by Leeming et al. [74], it was reported that Malassezia organisms were found in $68 \%$ of 3-day old acne papules. The report was documented over 20 years ago, but researchers only recently have attempted to target this organism in treatment $[4 \bullet \bullet$. The effects of azole antifungals on acne are multidimensional, as these medications are not only effective as antifungals but active against $P$. acnes [75••]. A study by Sugita et al. [75••] showed in vitro efficacy of miconazole, ketoconazole, and itraconazole against antibiotic-sensitive and antibiotic-resistant strands of $P$. acnes. Moreover, azole medications are known to have anti-inflammatory effects [76] that may further add to their effectiveness in acne therapy $[4 \cdot \bullet]$.

\section{Hormonal Antiandrogens in Acne}

It has long been known that the activation of androgen receptors leads to increased sebum production and plays a key role in acne pathogenesis. Oral contraceptives and spironolactone are the primary antiandrogenic treatments used in women with polycystic ovarian syndrome, hyperandrogenism, and premenstrual acne flares [60•]. Although spironolactone is not approved by the FDA for the treatment of acne, it is often prescribed off label [60•]. In the authors' opinion, adult females who present with acne primarily on the lower face respond well to spironolactone, even in the absence of hormonal dysregulation. Drospirenonecontaining oral contraceptives are also particularly effective in controlling acne, as drospirenone is a progestin with no androgenic capacity $[4 \bullet \bullet, 77]$.

Some topical hormonal agents show promise for the treatment of acne but are not yet available. Cortexolone $17 \propto$-propionate is a new steroidal antiandrogen topical cream under development for the treatment of acne. In a recent pilot randomized, double-blind study, cortexolone $17 \propto$-propionate was found to significantly reduce total lesion counts and inflammatory lesion counts to a greater extent than topical tretinoin $0.05 \%$ cream and placebo. [78]. Although this trial was limited in scope, it may stimulate future studies using topical antiandrogens in the treatment of acne. Another topical agent in development for the treatment of acne is nestorone, a nonandrogenic progestin combined with ethinyl estradiol $[4 \bullet \bullet, 79]$.

As mentioned earlier, melanocortins and melanocortin receptors (MC1R-MC5R) have been shown to play a role in sebogenesis [17-19]. Moreover, it is known that MC1R is expressed in several cell types of human skin, and MC5R is expressed in human sebaceous glands [17]. A novel MC1R and MC5R antagonist (JNJ-10229570) for the topical treatment of acne is currently being investigated. Results of a recent study of the topical MCR antagonist demonstrated marked reduction in sebum gland size and sebum-specific lipid production in human skin transplanted to mice with severe combined immunodeficiency $[80 \bullet \cdot$.

\section{A Changing Paradigm: Diet and Acne}

The link between diet and acne has presented much controversy over the years [81•]. Recently, researchers have revisited the link with particular attention to the role of dairy products (in particular skim milk) and high-glycemic load diets [81•]. Although the strongest evidence to date focuses on these two dietary influences, some preliminary studies suggest that antioxidants, fish oil, and probiotics may also play a role in acne $[61,82]$.

\section{Procedural Treatments for Acne}

Procedural treatments such as light and laser therapy are gaining popularity in the treatment of acne. These treatments are particularly attractive to patients as they represent cutting-edge alternatives to conventional acne treatments [83]. The following sections discuss some of the most recent research on these technologies. 
Lights and Lasers

P. acnes produces photoactive substances called porphyrins that when exposed to visible light produce reactive oxygen species that are toxic to the bacteria [84]. In addition to these antimicrobial effects, blue light has also been shown to have anti-inflammatory properties [85]. Recently, at-home devices using red or blue light have become popular among patients. A recent randomized self-control study of homeuse blue-light therapy demonstrated significant improvement in acne lesions as compared to placebo [86]. Photodynamic therapy is the technique of introducing exogenous porphyrins into the skin that can then be activated by light [83]. A recent study suggests this procedure works by downregulating TLR-2 and TLR-4 expression in the epidermis and sebaceous glands of patients with acne [87]. As discussed earlier, the activation of TLRs is thought to play a key role in the pathogenesis of acne [5].

\section{Photopneumatic Therapy}

Photopneumatic therapy is a device using negative pressure applied as suction with delivery of broadband, pulsed light. It is the only laser or light therapy approved by the FDA for acne [83]. The authors (Bowe and Shalita) have conducted the first RCT evaluating the efficacy of this therapy [88]. This study examined the effectiveness of Isolaz ${ }^{\mathbb{B}}$ (Solta Medical, Hayward, California), a photopneumatic device that combines vacuum pressure with a broadband light source (400-1,200 nm). Thirty-seven patients were randomized to receive vacuum therapy alone or in combination with broadband light. Inflammatory and noninflammatory lesions were decreased in both groups, with no significant difference between groups. The results indicate that both pneumatic therapy and photopneumatic therapy is effective in the treatment of acne. It is thought the mechanical disruption of biofilms in the PSU combined with evacuation of follicular contents is the primary mechanism of action of photopneumatic therapy.

\section{Conclusion}

Acne vulgaris is an extremely common skin condition, resulting in significant physical and emotional morbidity. Currently, the treatment of acne primarily includes the use of topical and oral medications. Fixed-dose topical drug combinations have been shown to be most effective in the treatment of mild to moderate acne, as they improve therapeutic efficacy, reduce side effects, and promote patient adherence. Although antibiotic treatment remains a key tool in acne therapy, increasing concerns regarding antibiotic resistance have lead dermatologists to reduce the dosage and length of antibiotic therapy. Additionally, new drug regimens that do not include antibiotic therapy are growing in popularity, including the use of nonantibiotic combination therapies, antifungal agents, and hormonal modulators. The role of diet has also become a popular discussion among dermatologists, with intriguing research demonstrating the negative effects of certain dairy products and highglycemic load diets on acne. Finally, research into procedural treatments for acne continues with the need for more clinical trials to truly elucidate the value of these therapies. As the field of dermatology continues to gain insights regarding acne pathogenesis and combine this knowledge with cutting-edge delivery mechanisms, options for treating acne will amplify.

Disclosure W. P. Bowe: consultant to Johnson \& Johnson, and payment for lectures from Johnson \& Johnson. J. B. Glick: none; A. R. Shalita: grants from Medicis and La Roche-Posay, consultant to Medicis and Galderma, travel support for study from Galderma, and minority shareholder in Medicis.

\section{References}

Papers of particular interest, published recently, have been highlighted as:

- Of importance

.• Of major importance

1. White GM. Recent findings in epidemiologic evidence, classification and subtypes of acne vulgaris. J Am Acad Dermatol. 1998;39: S34-7.

2. • Thiboutot D, Golllnick H, Bettoli V, et al. Global alliance to improve outcomes in acne. New insights into the management of acne: an update from the Global Alliance to Improve Outcomes in Acne group. J Am Acad Dermatol. 2009;60(5 Suppl):S1-S50. This article offers an important discussion of the Alliance's current stance on acne treatment.

3. Harper JC, Thiboutot DM. Pathogenesis of acne: recent research advances. Adv Dermatol. 2003;19:1-10.

4. •• Danby FW. New, relevant information and innovative interventions in the management of acne. G Ital Dermatol Venereol. 2011;146:197-210. This article provides the most up-to-date information on new concepts in acne pathophysiology and management.

5. Kim J, Ochoa MT, Krutzik SR, et al. Activation of toll-like receptor 2 in acne triggers inflammatory cytokine responses. J Immunol. 2002;169:1535-41.

6. Jeremy AH, Holland DB, Roberts SG, et al. Inflammatory events are involved in acne lesion initiation. J Invest Dermatol. 2003;121:20-7.

7. Burkart CG, Burkhart CN. Expanding the microcomedone theory and acne therapeutics: propionibacterium acnes biofilm produces biological glue that holds corneocytes together to for plug. J Am Acad Dermatol. 2007;57:722-4.

8. Melnik BC. Acneigenic stimuli coverage in phosphoinositol3 kinase/Akt/Fox01 signal tranduction. J Clin Exp Dermatol. 2010;101:1-8. 
9. $・$ Melnik BC. Fox01-the key for the pathogenesis and therapy of acne? J Dtsch Dermatol Ges. 2010;8:105-42. This article presents new concepts into the activation of the androgen receptor in the pathogenesis of acne.

10. Melnik BC. The role of transcription factor Fox01 in the pathogenesis of acne vulgaris and the mode of isotretinoin action. J Clin Exp Dermatol. 2010;145:559-72.

11. Melnik BC, Schmitz G. Role of insulin, insulin-like growth factor1 , hyperglycemic food and mlk consumption in the pathogenesis of acne vulgaris. Exp Dermatol. 2009;18:833-41.

12. •- Bellew S, Thiboutot D, Rosso JQD. Pathogenesis of acne vulgaris: what's new, what's interesting and what may be clinically relevant. J Drug Dermatol. 2011;10:582-5. This review provides excellent information on the most up-to-date developments in acne pathogenesis.

13. Slominiski A, Wortsman J, Luger T, et al. Cortiocotropin releasing hormone: and propiomelanocortin involvement in the cutaneous response to stress. Physiol Rev. 2000;15:2291-9.

14. Kono M, Nagata H, Umemura S, et al. In situ expression of corticotrophin-releasing hormone (CRH) and proopiomelanocortin (POMC) genes in human skin. FASEB J. 2001;15:2297-9.

15. Zouboulis CC, Seltmann H, Hiroi N, et al. Corticotrophin releasing hormone: an autocrine hormone that promotes lipogenesis in human sebocytes. Proc Natl Acad Sci USA. 2002;99:7148-53.

16. Krause K, Schnitger A, Fimmel S, et al. Corticotrophinreleasing hormone skin signaling is receptor-medicated and is predominant in the sebacous glands. Horm Metab Res. 2007;38:166-70

17. Zhang L, Li WH, Anthonavage M, et al. Melanocortin-5 receptor and sebogenesis. Eur J Pharmacol. 2011;660:202-6.

18. Bohm M, Schiller M, Stander S, et al. Evidence of experssion of melanocortin-1 receptor in human sebocytes in vitro and in situ. $\mathrm{J}$ Invest Dermatol. 2002;118:533-9.

19. Gancevicience R, Graziene V, Bohm M, et al. Increased in situ expression of melanocortin-1 receptor in sebacous glands of lesional skin of patients with acne vulgaris. Exp Dermatol. 2007;16:547-52.

20. Gollnick H, Cunliffe W, Berson D, et al. Management of acne: a report from a Global Alliance to Improve Outcomes in Acne. J Am Acad Dermatol. 2003;49(1 Suppl):S1-S37.

21. Webster GF. Topical tretinoin in acne therapy. J Am Acad Dermatol. 1998;39:S38-44.

22. Thielitz A, Gollnick H. Topical retinoids in acne vulgaris: efficacy and safety. Am J Clin Dermatol. 2008;9:369-81.

23. Gans EH, Kligman AM. Comparative efficacy of clindamycin and benzoyl peroxide: in-vivo suppression of Propionbacterium acnes. J Dermatol Treat. 2002;13:107-10.

24. Leyden JJ, Wortzman M, Baldwin EK. Antbiotic-resistance Propinibacterium acnes suppressed by a benzoyl peroxide cleanser $6 \%$. Cutis. 2010;82:417-21.

25. Leyden JJ, Kaidbey K, Levy SF. The combination formulation of clindamycin $1 \%$ plus benzoyl peroxide $5 \%$ versus 3 different formulations of topical clindaymycin alone in the reduction of Propionbacterium acnes: An in-vivo comparative study. Am J Clin Dermatol. 2001;2:263-6.

26. - Yentzer BA, Ade RA, Fountain JM, et al. Simplifying regimens promotes greater adherence and outcomes with topical acne medications: a randomized controlled trial. Cutis. 2010;86:103-8. This is a well-designed clinical study demonstrating the effectiveness of fixed-drug combination therapies on patient adherance in the treatment of acne vulgaris.

27. Mordon S, Sumian C, Devoisselle JM. Site-specific methylene blue delivery to pilosebacous structures using highly porous nylon microspheres: an experimental evaluation. Lasers Surg Med. 2003;33:119-25.
28. Vogt A, Combadiere B, Hadam S, et al. $40 \mathrm{~nm}$, but not $750 \mathrm{~nm}$ or $1,500 \mathrm{~nm}$, nanoparticles enter epidermal CD1 a + cells after transcutaneous application on human skin. J Am Acad Dermatol. 2006; 126:1316-22.

29. Jung $\mathrm{S}$, Otberg $\mathrm{N}$, Thiede $\mathrm{G}$, et al. Innovative liposomes as a transfollicular drug delivery system: penetration into porcine hair follicles. J Invest Dermatol. 2006;126:1728-86.

30. - Castro GA, Ferreira LA. Novel vesicular and particular drug delivery systems for topical treatment of acne. Expert Opin Drug Deliv. 2008;5. This article provides a well-constructed overview of novel drug delivery systems.

31. Honzak L, Sentjurc M. Development of liposome encapsulated clindamycin for treatment of acne vulgaris. Pflugers Arch. 2000;440:44-5.

32. Smith S, Morhenn V, Webster G. The characteristics and utility of solid phase porous microspheres: a review. J Drugs Dermatol. 2006;5:969-74.

33. Nighland M, MY M, Wisniewski S, et al. The effect of simulated solar UV irradiation on tretinoin in tretinoin gel microsphere $0.1 \%$ and tretinoin gel $0.025 \%$. Cutis. 2006; 77:313-6.

34. Nyirady JLC, Yusuf M, et al. The stability of tretinoin in tretinoin gel microsphere $0.1 \%$. Cutis. 2002;70:295-8.

35. Berger R, Rizer R, Barba A, et al. Tretinoin gel microspheres $0.04 \%$ versus $0.1 \%$ in adolescents and adults with mild to mocerate acne vulgaris: a 12 week, multicenter, randomized, double-blind, parallel-group, phase IV trial. Clin Ther. 2007;29:1086-97.

36. Leyden JJ, Nighland M, Rossi AB, et al. Irritation potential of tretinoin gel microsphere pump versus adapalene plus benzoyl peroxide gel. J Drug Dermatol. 2010;9:998-1003.

37. Smith SR, Kempers S. A study of $5.5 \%$ benzoyl peroxide microsphere cream as monotherapy versus $6 \%$ benzoyl peroxide gel in the treatment of acne vulgaris. Cosmet Dermatol. 2006;19:537-42.

38. - Rosso JQD. Benzoyl peroxide formulations: what is the science supporting microsphere vehicle technology and clincal use? J Clin Aesthet Dermatol. 2009;2:46-54. This article provides an excellent framework for the discussion of microsphere technology and its relationship to BPO products in the treatment of acne.

39. Nyirady J, Nighland M, Payonk G, et al. A comparative evaluation of tretinoin gel microsphere, $0.1 \%$ versus tretinion cream, $0.025 \%$ in reducing facial shine. Cutis. 2000;66:153-6.

40. Date AA, Joshi MD, Patravale VB. Parasitic diseases: liposomes and polymeric nanoparticles versus lipid nanoparticles. Adv Drug Deliv Rev. 2006;59:505-21.

41. - Castro G, Oliveira C, Mahecha C, et al. Comedolytic effect and reduced skin irritation of a new formulation of all-trans retinoic acid-loaded solid lipid nanoparticles for topical treatment of acne. Arch Dermatol Res. 2011;303:513-20. This is an interesting study of atRA-loaded SLNS for the treatment of acne.

42. Ridolfi DM, Marcato PD, Justo GZ, et al. Chitosan-solid lipid nanoparticles as carriers for topical delivery of tretinoin. Colloids Surf B Biointerfaces. 2012;93:36-40.

43. Torok HM, Pillai R. Safety and effiacy of micronized tretinoin gel $(0.05 \%)$ in treating adolescent acne. J Drug Dermatol. 2011;10:647-51.

44. Michael G, Truquet M, Mondain J, et al. Comparison of in vivo antibacterial actiity of hydro-alcoholic solutions of hexamidine and chlorhexidine. J Int Med Res. 1986;14:205-9.

45. Lee HE, Ko JY, Kim YH, et al. A double-blind randomized controlled comparison of APDDR-0901, a novel cosmeceutical formulation, and.1\% adapalene gel in the treatment of mild to moderate acne vulgaris. Eur J Dermatol. 2011;21:959-65. 
46. Leyden JJ. Efficacy of benzoyl peroxide (5.3\%) emollient foam and benzoyl peroxide ( $8 \%$ ) Wash in reducing propionibacterium acnes on the back. J Drug Dermatol. 2010;9:622-5.

47. Tan HH. Antibacterial therapy for acne: a guide to selection and use of systemic agents. Am J Clin Dermatol. 2003;4:307-14.

48. Strauss JS, Krowchuk DP, Leyden JJ, et al. Guidelines for care for acne vulgaris management. J Am Acad Dermatol. 2007; 56:651-63.

49. Mouser PE, Baker BS, Seaton ED, et al. Propionbacerium acnesreactive T helper- 1 cells in the skin of patients with acne vulgaris. $\mathrm{J}$ Invest Dermatol. 2003;121:1226-8.

50. Sapadin AN, Fleischmajer R. Tetracyclines: nonantibioitic properties and their clinical implications. J Am Acad Dermatol. 2006;54:133-5.

51. Webster G, Rosso JQD. Anti-inflammatory activity of tetracyclines. Dermatol Clin. 2007;25:133-5.

52. Rosso JQD, Kim G. Optimizing use of oral antibiotics in acne vulgaris. Dermatol Clin. 2009;27:33-42.

53. Plott RT, Wortzman MS. Key bioavailability features of a new extended-release formulation of minocycline hydrochloride tablets. Cutis. 2006;78:6-10.

54. - Ochsendorf F. Minocycline in acne vulgaris benefis and risks. Am J Clin Dermatol. 2010;11:327-41. This is an excellent review of the effects of minocycline in the treatment of acne vulgaris.

55. Eady EA, Cove JH, Holland KT, et al. Superior antibacterial action and reduced incidence of bacterial resistance in minocycline compared to tetracycline-treated acne patients. $\mathrm{Br} \mathrm{J}$ Dermatol. 1990;122:233-44.

56. Eady EA, Jones CE, Gardner KJ, et al. Tetracycline-resistant propionibacteria from acne patients are cross-resistant to doxycycline, but sensitive to minocycline. Br J Dermatol. 1993;128:556-60.

57. Fleischer AB, Dinehart S, Stough D, et al. Safety and efficacy of a new extended-release formulation of minocycline. Cutis. 2006;78:21-31.

58. Rosso JD. Clinical significance of brand versus generic formulations: focus on oral minocyline. Cutis. 2006;77:153-6.

59. Stewart DM, Torok HM, Weiss JS. Dose-ranginig efficacy of new once-daily extended-release minocycline for acne vulgaris. Cutis. 2006;78 suppl 4:11-20.

60. - Newman M, Bowe WP, Shalita AR. Therapeutic considerations for severe nodular acne. Am J Clin Dermatol. 2011;12:7-14. This article offers a well-written discussion of therapeutic options for severe acne.

61. Bowe W, Logan AC. Acne vulgaris, probiotics and the gut-brainskin axis - back to the future? Gut Pathogens. 2011;3:1-11.

62. • Patel M, Bowe WP, Heughebaert A, et al. The development of antimicrobial resistance due to the antibiotic treatment of acne vuglaris: a review. J Drug Dermatol. 2010;2010:655-64. This excellent review focuses on the effects of antimicrobial resistance resulting from antibiotic therapy of acne vulgaris.

63. Margolis DJ, Bowe WP, Hoffstad O, et al. Antibiotic teatment of acne may be associated with upper respiratory tract infections. Arch Dermatol. 2005;215:213-8.

64. Margolis DJ, Fanelli M, Kupperman E, et al. Association of pharyngitis with oral antibiotic use for the treatment of acne: a cross-sectional and prospective cohort study. Arch Dermatol. 2011;Epub ahead of print.

65. Mills O, Thornsberry C, Cardin CW, et al. Bacterial resistance and therapeutic outcome following three months of topical acne therapy with $2 \%$ erythromycin gel versus its vehicle. Acta Derm Venereol. 2002;82:260-5.

66. Vowels BR, Feingold DS, Sloughfy C, et al. Effects of topical erythromycin on ecology of aerobic cutaneous bacterial flora. Antimicob Agents Chemother. 1996;40:2598-604.
67. Miller YW, Eady EA, Lacey RW, et al. Sequential antibiotic therapy for acne promotes carriage of resistant staphylococci on the skin of contacts. J Antimicrob Chemother. 1996;38: 829-37.

68. Naidoo J. Interspecific co-transfer of antibiotic resistance plasmids in staphylococci in vivio. J Hyg (Lond). 1984;93:59-66.

69. - Leyden JJ, Preston N, Osborn C, et al. In-vivo effectiveness of adapalene $0.1 \%$ /benzoyl peroxide $2.5 \%$ gel on antibiotic-sensitive and resistant propionibacterium acnes. J Clin Aesthet Dermatol. 2011;4:22-6. This is an important study demonstrating the effectivess of a nonantibiotic topical combination therapy on antibioticresistant $\mathrm{P}$ acnes.

70. - Stotland M, Shalita AR, Kissling RF. Dapsone $5 \%$ : a review of its efficacy and safety in the treatment of acne vulgaris. Am J Clin Dermatol. 2009;10:221-7. This is an excellent, comprehensive review on the clinical studies evaluating the safety and efficacy of dapsone gel.

71. Draelos ZD, Carter E, Maloney JM, et al. Two randomized studies demonstrate the effiacy and safety of dapsone gel, $5 \%$ for the treatment of acne vulgaris. J Am Acad Dermatol. 2007;56:439-47.

72. Lucky AW, Maloney JM, Roberts J, et al. Dapsone gel $5 \%$ for the treatment of acne vulgaris: safety and efficacy of longer (1 year) treatment. J Drug Dermatol. 2007;6:981-7.

73. Thiboutot DM, Willmer J, Sharata H, et al. Pharmacokinetics of dapsone gel $5 \%$ for the treatment of acne vulgaris. J Clin Pharmacokinet. 2007;46:697-712.

74. Leeming JP, Holland KT, Cuncliffe WJ. The microbial colonization of inflamed acne vulgaris lesions. Br J Dermatol. 1988;1188:203-8.

75. •- Sugita T, Miyamoto M, Tsuboi R, et al. In vitro activities of azole antifungal agents against Propionibacterium acnes isolated from patients with acne vulgaris. Biol Pharm Bull. 2010;33:125-7. This article documents some of the first studies demonstrating the effectiveness of antifungal therapy for acne vulgaris.

76. Sur R, Babad JM, Garay M, et al. Anti-inflammatory activity of setraconazole nitrate is mediated via activitation of a p38-COX-2PGE2 pathway. J Invest Dermatol. 2008;128:336-44.

77. Rubig A. Drospirenone: a new cardiovascular-active progestin with antialdoesterone and antiandrogenic properities. Climacteric. 2003;6:49-54.

78. Tifu V, Tiplica GS, Naumescu E, et al. Cortexolone 17 alphapropionate $1 \%$ cream, a new potent antiandrogen for topical treatment of acne vulgaris. A pilot randomized, double blind comparative study vs. placebo and tretinoin. $05 \%$ cream. Br J Dermatol. 2011;165:177-83.

79. Kumar N, Koide SS, Tsong Y, et al. Nestorone: a progestin with a unique pharmacological profile. Steroids. 2000;65:629-36.

80. •- Eisinger M, Li W-H, Anthonavage M, et al. A melanocortin receptor 1 and 5 antagonist inhibits sebaceous gland differentiation and the production of sebum-specific lipids. J Dermatol Sci. 2011;63:23-32. This important study focuses on the role of melanocortin receptors in sebogenesis.

81. - Bowe WP, Koshi S, Shalita AR. Diet and acne. J Am Acad Dermatol. 2010;63:124 41. This article provides a historical framework and current information on the influence of diet on acne.

82. Bowe WP, Logan AC. Clinical implications of lipid peroxidation in acne vulgaris: old wine in new bottles. Lipids Health Dis. 2010;9:141.

83. Bowe WP, Shalita AR. Procedural treatments for acne. In: Shalita AR, Rosso JQD, Webster GF, editors. Acne vulgaris. New York: Informa Healthcare; 2011. p. 208-17.

84. Lee WL, Shalita AR. Comparative studies of porphyrin production in Propionibacterium acnes and Propionibacterium granulosum. J Bacteriol. 1978;133:811-5.

85. Shnitkind E, Yaping E, Geen S, et al. Anti-inflammatory properties of narrow-band blue light. J Drug Dermatol. 2006;5:605-10. 
86. Gold MH, Sending W, Biron JA. Clinical efficacy of home-use blue light therapy for mild-to moderate acne. J Cosmet Laser Ther. 2011;12:308-14.

87. Jeong E, Hong JW, Min JA, et al. Topical ALA-photodynamic therapy for acne can induce apoptosis of sebocytes and downregulate their TLR-2 and TLR-4 expression. Ann Dermatol. 2011;23:23-32.

88. Bowe W, Heughebaert C, Doyle A, et al. Pilot study of vacuum versus vacuum plus light in treatment of acne. Poster presentation at 2011 MauiDerm Conference, Maui, February 23-27, 2011.

89. Draelos ZD, Potts A, Saenz ABA. Randomized tolerability analysis of clindamycin phosphate $1.2 \%$-tretinoin $0.025 \%$ gel used with benzoyl peroxide wash $4 \%$ for acne vulgaris. Cutis. 2010;86:210-8.

90. Babayeva L, Akarsu S, Fetil E. Comparison of tretinoin $0.05 \%$ cream and $3 \%$ alcohol-based salicylic acid preparation in the treatment of acne vulgaris. J Eur Acad Dermatol Venereol. 2011;25:328-33.

91. Pazoki-Toroudi H, Nilforoushzadeh MA, Ajami M, et al. Combination of azelaic acid $5 \%$ and clindamycin $2 \%$ for the treatment of acne vulgaris. Cutan Ocul Toxicol. 2011;30:286-91.

92. Poulin Y, Sanchez NP, Bucko A, et al. A 6-month maintenance therapy with adapalene-benzoyl peroxide gel prevents relapse and continuously improves efficacy among patients with severe acne vulgaris: results of a randomized controlled trial. $\mathrm{Br} \mathrm{J}$ Dermatol. 2011;1376-82.

93. Tanghetti E, Dhawan S, Green L, et al. Clinical evidence for the role of a topical anti-inflammatory agent in comedonal acne: findings from a randomized study of dapsone gel $5 \%$ in combination with tazarotene cream $0.1 \%$ in patients with acne vulgaris. J Drugs Dermatol. 2011;10:783-92.

94. Wilhelm K, Wilhelm D, Neumeister C, et al. Lack of irritative potential of nadifloxacin $1 \%$ when combined with other topical anti-acne agents. Clin Exp Dermatol. 2011; [Epub ahead of print].

95. Choudhury S, Chatterjee DK, Sarkar DK, et al. Efficacy and safety of topical nadifloxacin and benzoyl peroxide versus clindamycin and benzoyl peroxide in acne vulgaris: a randomized controlled trial. Indian J Pharmacol. 2011;43:628-31.

96. Schmidt N, Gans H. Clindamycin $1.2 \%$ tretinoin $0.025 \%$ gel versus clindamycin gel treatment in acne patients a focus on fitzpatrick skin types. J Clin Aesthet Dermatol. 2011;4:31-40.

97. Eichenfield LF, Sáenz ABA. Safety and efficacy of clindamycin phosphate $1.2 \%$-benzoyl peroxide $3 \%$ fixed-dose combination gel for the treatment of acne vulgaris: a phase 3, multicenter, randomized, double-blind, active- and vehicle-controlled study. J Drugs Dermatol. 2011;10:1382-96.

98. Kobayashi M, Nakagawa T, Fukamachi K, et al. Efficacy of combined topical treatment of acne vulgaris with adapalene and nadifloxacin: a randomized study. J Dermatol. 2011;38:1163-3. 